\title{
Species-independent bioassay for sensitive quantification of antiviral type I interferons
}

\author{
Thomas Kuri, Matthias Habjan, Nicola Penski, Friedemann Weber ${ }^{*}$
}

\begin{abstract}
Background: Studies of the host response to infection often require quantitative measurement of the antiviral type I interferons (IFN- $\alpha / \beta)$ in biological samples. The amount of IFN is either determined via its ability to suppress a sensitive indicator virus, by an IFN-responding reporter cell line, or by ELISA. These assays however are either time-consuming and lack convenient readouts, or they are rather insensitive and restricted to IFN from a particular host species.

Results: An IFN-sensitive, Renilla luciferase-expressing Rift Valley fever virus (RVFV-Ren) was generated using reverse genetics. Human, murine and avian cells were tested for their susceptibility to RVFV-Ren after treatment with species-specific IFNs. RVFV-Ren was able to infect cells of all three species, and IFN-mediated inhibition of viral reporter activity occurred in a dose-dependent manner. The sensitivity limit was found to be $1 \mathrm{U} / \mathrm{ml}$ IFN, and comparison with a standard curve allowed to determine the activity of an unknown sample.

Conclusions: RVFV-Ren replicates in cells of several species and is highly sensitive to pre-treatment with IFN. These properties allowed the development of a rapid, sensitive, and species-independent antiviral assay with a

convenient luciferase-based readout.
\end{abstract}

\section{Background}

Type-I interferons (IFN- $\alpha / \beta)$ are potent cytokines that can be released from virtually all vertebrate cells following viral infection. They comprise a large number of IFN- $\alpha$ subspecies and a single IFN- $\beta$, and their actions reflect an important part of the innate immune system [1]. Upon infection, viruses are detected by one or several different pattern recognition receptors and production of IFN is induced. Newly synthesized IFNs are secreted in order to bind to their specific receptor (which is common for IFN- $\alpha$ and IFN- $\beta$ ) in an autocrine and paracrine manner. Receptor signaling via the Jak/Stat pathway leads to the up-regulation of a set of IFN-stimulated genes (ISGs), some of which having antiviral activity. As a consequence, neighbouring cells establish an antiviral state to prevent viral spread in the organism [2].

A wide variety of assays has been developed to determine the presence and activity of antiviral IFNs [3]. One type of assay is based on the upregulation of ISGs, either directly by measuring enzymatic ISG products [4], or

\footnotetext{
* Correspondence: friedemann.weber@uniklinik-freiburg.de

Department of Virology, University of Freiburg, D-79008 Freiburg, Germany
}

indirectly by using cells containing a reporter gene under control of an IFN-responsive promoter. Often, the promoter of the Mx gene is used [5-8] due to the sensitivity and the low background expression of this ISG [9]. Although cell-line based ISG/reporter assays are rather convenient, a major drawback is their restriction to a particular host organism since IFNs bind to their receptor in a species-specific manner. In a similar vein, commercially available ELISAs are limited to a particular type of IFN and a single host species.

The historically oldest and still widely used assay to determine IFN activity are assays of antiviral activity. Here, IFN-mediated protection of cells is directly analyzed after infection with a sensitive challenge virus. The presence of IFNs is reflected by reduced cytopathic effects or diminished viral growth [10,11]. Some recent modifications of this assay take advantage of green fluorescent protein (GFP)-expressing viruses. These viruses allow to determine the reduction in virus titers by counting GFP-positive cells, either manually or by flow cytometry [12-14].

Rift Valley fever virus (RVFV) is a highly pathogenic member of the family Bunyaviridae. RVFV encodes a 
non-structural gene termed NSs which is mainly responsible for the pathogenicity of this virus $[15,16]$. Mutant RVFV lacking the NSs gene are thus highly sensitive to IFN-induced antiviral proteins such as MxA and PKR [17-20]. Here, we applied our recently developed reverse genetics system for RVFV [21] and replaced the NSs gene with the Renilla luciferase reporter gene, resulting in an attenuated, IFN-sensitive virus. We used this virus to establish a bioassay for quantification of IFN that combines the advantages of the classical antiviral assay with the convenience of luciferase reporter assays.

\section{Results and discussion}

Establishment of a luciferase-based antiviral IFN assay

Using our reverse genetics system [21], we generated the recombinant virus RVFV-Ren with the IFN antagonist NSs replaced by Renilla luciferase (see Materials and Methods). Luciferase expression by this virus was correlating with viral replication and remained stable over several passages (data not shown). We then tested the IFN sensitivity of RVFV-Ren in human A549 cells. Cells were seeded in 96-well microtiter plates and pre-treated with serial dilutions of standard IFN for 7 hours to allow the establishment of an antiviral state. Afterwards, cells were infected with RVFV-Ren at an MOI of 1 for 16 hours and Renilla luciferase activity was measured in cell lysates (Fig. 1A). To determine the linear range of the assay, IFN dilutions from $0.5 \mathrm{U} / \mathrm{ml}$ up to $100 \mathrm{U} / \mathrm{ml}$ were tested. A significant reduction of luciferase activity was observed using IFN concentrations from $1 \mathrm{U} / \mathrm{ml}$ on (Fig. 1B). The highest concentration of IFN in the linear range was $50 \mathrm{U} / \mathrm{ml}$.

\section{Measurement of IFN in biological samples}

We used the RVFV-Ren assay to quantify IFN induction by two recombinant viruses which had been generated by our group. Wild-type (wt) La Crosse virus is able to suppress IFN induction, whereas a mutant virus upregulates the IFN- $\beta$ gene [22]. Supernatants were taken from cells infected with these viruses and sterilized with $\beta$ propiolactone to destroy infectiousness. After removal of the disinfectant, undiluted and serially diluted samples of supernatants were subjected to the RVFV-Ren assay in parallel to the serial dilutions of standard IFN (see Fig. 1B). The IFN dilutions were used to create a standard curve by regression analysis (Fig. 2A) which, in turn, served to calculate IFN concentrations of the La Crosse virus supernatants. For cells infected with wt La Crosse virus, the activity of the undiluted supernatants was taken to determine the amount of type I IFN. For the mutant virus, we had to use the 1:10 dilution as the basis for the calculations since undiluted supernatants would have been out of the linear range of the assay. As expected, only little IFN was present in supernatants of cells infected with wt La Crosse virus, but substantial amounts were measured in the supernatant of mutant virus-infected cells (Fig 2B).

\section{A}

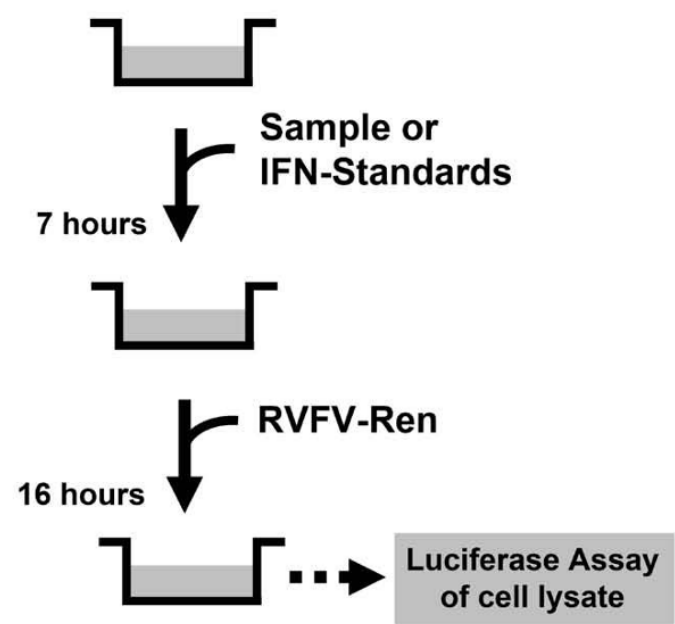

B



Figure 1 Principle and evaluation of the RVFV-Ren antiviral assay. (A) Schematic outline of the RVFV-Ren antiviral assay. (B) Dose-response curve in human A549 cells. A549 cells seeded in 96-well plates were treated with increasing doses of Multiferon for 7 hours before infection with 10,000 plaque forming units of RVFV-Ren. Sixteen hours later, cells were lysed and Renilla luciferase activity in cell lysates was determined. All measurements were performed in triplicate wells under standard conditions; shown are means $\pm S D$. Both axes are plotted in logarithmic scale; the dotted vertical line indicates the threshold of sensitivity, based on the mean + 2SD of an untreated control run in triplicate; shaded area shows the linear range of the assay; RLU, relative light units. 


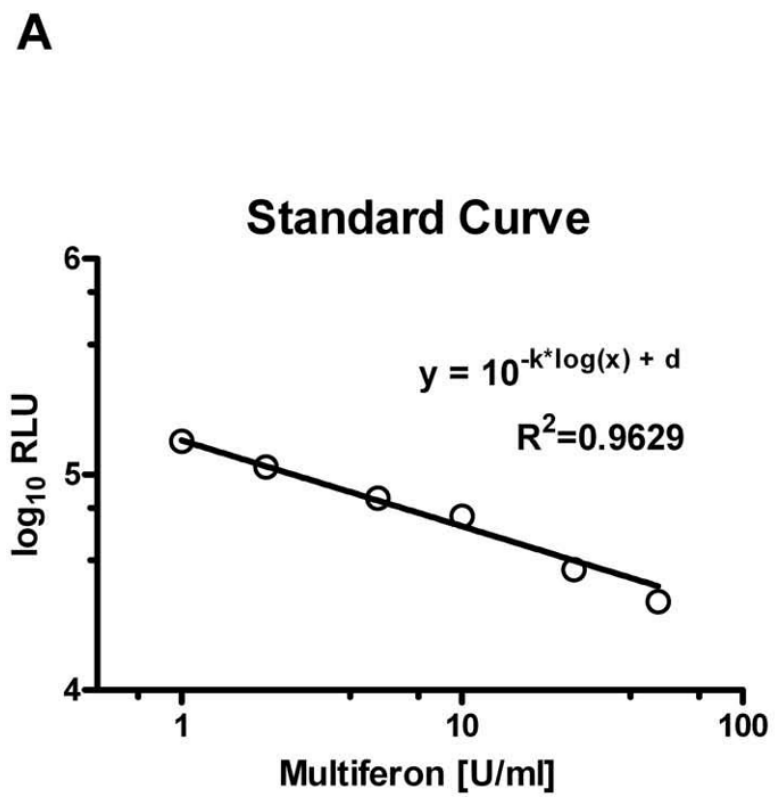

B

Figure 2 Measurement of IFN in samples by the RVFV-Ren antiviral assay. (A) Generation of the standard curve. All data points from Figure 1B which are above the sensitivity threshold and within the linear range of the assay (from 1-50 U/ml) are plotted. Non-linear regression analysis was performed to generate a standard curve and to compute the regression equation. Values for the slope $(\mathrm{k})$ and the $y$-axis interception point (d) were -0.3988 and 5.159, respectively. The coefficient of regression $\left(R^{2}\right)$ indicates the linearity of detection. (B) Analysis of two samples with low and high concentration of IFN. Supernatants from human A549 cells infected with wt mutant La Crosse virus were first sterilized with $\beta$ propiolactone (see Methods section) and then analyzed for inhibition of RVFV-Ren. Sterilized supernatant from uninfected cells served as control (mock). The IFN content of all samples was extrapolated from the standard curve shown in (A). All measurements were performed in triplicate; columns show means $\pm S D$, with actual values given above each column.

\section{Sensitive detection of murine and avian IFNs}

Since RVFV is able to infect a broad range of vertebrate hosts, we wanted to know whether the assay which was established for human IFN could be adapted to measure IFN from other species. To this aim, we infected L929 mouse cells and chicken embryo fibroblasts (CEFs), and tested the anti-RVFV-Ren activity of murine and avian IFN, respectively. As shown in Figure 3, RVFV-Ren was able to replicate in both cell lines and viral growth was inhibited in a dose-dependent manner by the speciescompatible IFN. The sensitivity of the assay on L929 (Fig. 3A) as well as on CEFs (Fig. 3B) was comparable to human cells, with a linear range from 1 to $50 \mathrm{U} / \mathrm{ml}$ and from 1 to $25 \mathrm{U} / \mathrm{ml}$, respectively.

\section{The RVFV-Ren assay measures type I IFNs}

Type I IFNs are the main, but not the only, cytokines produced during an innate immune response. We wanted to know whether other antiviral molecules, e.g. IFN- $\lambda$, could disturb our bioassay. To investigate this, we employed embryo fibroblasts from knockout mice lacking the receptor for type I IFNs (IFNAR-/- MEFs). Supernatants containing antiviral mouse cytokines were obtained by infecting L929 cells with the RVFV strain clone $13[15,16]$, a virus mutant which in our experience is one of the strongest inducers of antiviral cytokines. Clone 13 upregulated an innate immune response including IFN- $\beta$, as expected (Fig. 4A). When we performed the bioassay on IFNAR-/- MEFs, the indicator virus RVFV-Ren was not inhibited by IFN- $\alpha$, as expected, but also not by supernatants (Fig. 4B). This strongly indicates that it is type I IFNs which are causing the antiviral effect against RVFV-Ren.

Similar to the conventional virus inhibition or GFPvirus-based IFN bioassays, it can not be excluded $a$ priori that material derived from e.g. infected dendritic cells or clinical samples may contain antiviral cytokines other than type I IFNs which could inhibit RVRV-Ren. Depending on the species material, IFNAR -/- MEFs, other mutant cell lines, acid treatment, or IFNAR-neutralizing antisera could be employed to verify that only type I IFNs are causing RVFV-Ren inhibition. However, in many cases supernatants from tissue cells are used, a system in which apparently type I IFNs are the dominant antiviral cytokine.

\section{Conclusions}

Here we established a novel antiviral bioassay based on a recombinant RVFV encoding the gene for Renilla luciferase in place of the IFN-antagonistic NSs gene 


\section{A}

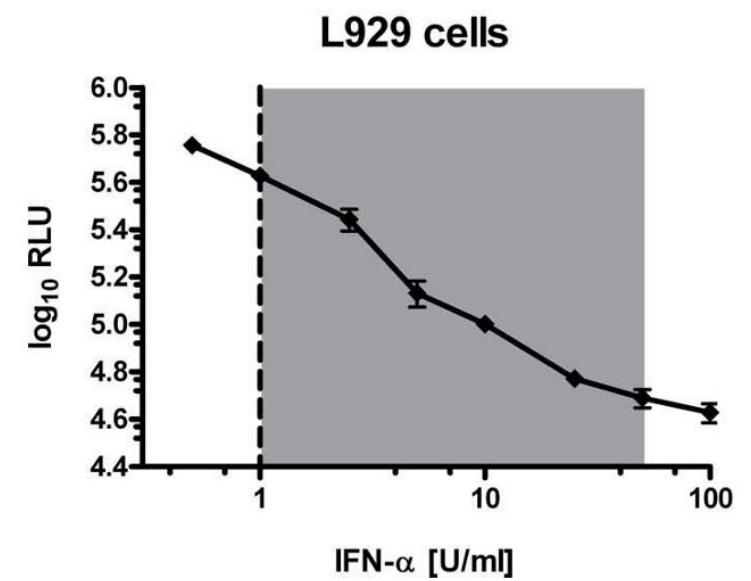

B



Figure 3 Dose-response curves for mouse and chicken IFNs. Murine L929 cells (A) or CEFs (B) seeded in 96-well plates were pre-treated for seven hours with increasing doses of pan-species IFN- $\alpha$ and chicken IFN (chIFN), respectively. Both assays were then carried out further as described for Fig. 1B. All measurements were performed in triplicate wells under standard conditions; shown are means \pm SD. Both axes are plotted in logarithmic scale; the dotted vertical line indicates the threshold of sensitivity, based on the mean $+2 S D$ of an untreated control run in triplicate; shaded area shows the linear range of the assay.

\section{A}

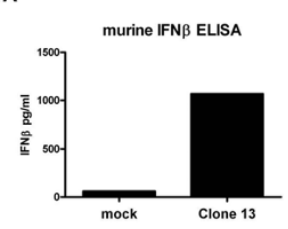

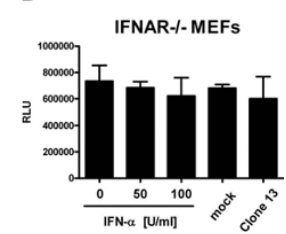

Figure 4 RFVFV-Ren antiviral assay on cells lacking the type I IFN receptor. Murine L929 cells were infected with Clone 13 at an $\mathrm{MOI}$ of 1 , or left uninfected (mock). After $16 \mathrm{~h}$ of infection, supernatants were harvested and sterilized as described for Fig. 2B. (A) Determination of IFN- $\beta$ using a commercial ELISA. Mean values from two measurements are shown. (B) Analysis for inhibition of RVFV-Ren on IFNAR-/- MEFs. Different doses of recombinant panspecies IFN- $\alpha$ were used as control. Measurements were performed in triplicate; columns show means \pm SD.

(RVFV-Ren). Growth of this virus is inhibited by IFN in a dose-dependent manner, which can easily be monitored by the measurement of luciferase activity in lysates of infected cells. Furthermore, IFN of at least three different species was reliably measured, indicating that RVFV-Ren not only infects cells of human or murine origin, as previously known, but also bird cells. The latter fact indicates that the RVFV-Ren assay could be used for other, less established species as well, e.g. bats. Our results show that these properties make this virus a useful tool for quantitative, species-independent measurement of IFN in biological samples, and that the use of luciferase and the 96-well plate format greatly facilitates readout. RVFV is classified as a BSL3 pathogen,

and RVFV-Ren also needs to be handled under BSL3 conditions. The principle of our assay, however, namely the use of an IFN-sensitive virus expressing Renilla luciferase might as well be adapted for IFN-sensitive nonBSL3 viruses with a broad host range such as Vesicular stomatitis virus or Newcastle disease virus.

\section{Methods}

\section{Cells and viruses}

BHK-21, Vero E6 (ATCC; CRL-1586), 293T (ATCC; CRL-11268), human A549 (ATCC; CCL-185) cells, and murine L929 cells (ATCC; CCL-1) and IFNAR -/- MEFs (kindly provided from Jovan Pavlovic, University of Zurich, Switzerland) were cultivated in Dulbecco's modified Eagle's medium (DMEM) supplemented with 10\% fetal calf serum (FCS; Biochrom AG) and antibiotics. Chicken embryo fibroblasts (CEF) were prepared from 10-day-old chicken embryos and maintained in DMEM with $2 \%$ chicken serum (Invitrogen), 8\% FCS and antibiotics. Viruses used in this study were recombinant Rift Valley fever virus (RVFV) expressing Renilla luciferase (see below), RVFV Clone 13 [15,16], and recombinant La Crosse viruses expressing (wt) or lacking expression (mutant) of the NSs gene [22]. Virus titers were determined by standard plaque assay on Vero E6 cells.

\section{Interferons}

Recombinant pan-species IFN- $\alpha$ (IFN- $\alpha$ B/D BglII) was purchased from PBL Biomedical Laboratories, and Multiferon, a mix of natural human IFN- $\alpha$ subtypes, was from Viragen. Recombinant chicken interferon (chIFN) 
was purified from E. coli and calibrated as described previously $[23,24]$.

\section{Generation of recombinant RVFV expressing Renilla luciferase}

We used our plasmid-based rescue system for generation of recombinant RVFV[21]. Expression of Renilla luciferase by RVFV-Ren was achieved by replacing the non-structural NSs gene on the genomic $S$ segment with the Renilla gene. The corresponding $\mathrm{S}$ segment rescue plasmid pHH21-RVFV-vN_Ren was generated by inserting the Renilla luciferase open reading frame, amplified from plasmid pRL-SV40 (Promega), into the cloning site of pHH21-RVFV-vN_TCS [21]. RVFV-Ren was rescued by transfecting cocultures of 293T and BHK-21 cells in six-well plates with $0.5 \mu \mathrm{g}$ of helper plasmids (pI.18-RVFV-L and pI.18-RVFV-N), together with $1 \mu \mathrm{g}$ each of pHH21-RVFV-vL, pHH21-RVFV-vM, and pHH21-RVFV-vN_Ren using Nanofectin transfection reagent (PAA Laboratories). Supernatants containing recombinant viruses were collected 5 days post transfection and used to grow virus stock on Vero E6 cells. All RVFV rescues were performed under biosafety level (BSL) 3 conditions.

\section{Antiviral bioassay}

Prior to measurement of IFN-containing samples, remaining virus was inactivated using $\beta$-propiolactone (Acros Organics) $[11,25]$. Briefly, supernatants were first incubated in the presence of $0.05 \% \beta$-propiolactone in plastic dishes overnight at $4{ }^{\circ} \mathrm{C}$, and then at $37^{\circ} \mathrm{C}$ for 2 hours for hydrolysis of $\beta$-propiolactone.

Approximately 10,000 cells were seeded into each well of a 96-well microtiter plate and incubated overnight in a humified incubator at $5 \% \mathrm{CO}_{2}$ and $37^{\circ} \mathrm{C}$. Cells were then treated either with different dilutions of standard IFN or serial ten-fold dilutions of IFN-containing samples in $100 \mu \mathrm{l}$ of growth medium for 7 hours. Subsequently, cell culture medium was removed and 10,000 plaque forming units of RVFV-Ren in $100 \mu$ l of infection medium (DMEM with 2\% FCS and 20 mM HEPES, pH 7.3) were added per well. After 16 hours of further incubation, supernatants were removed and cells lysed in 50 $\mu \mathrm{l}$ of $1 \times$ Renilla lysis buffer (Promega). Luciferase activity in $10 \mu \mathrm{l}$ of cell lysate was measured using the Renilla luciferase assay system (Promega), according to the manufacturer's instructions.

\section{List of abbreviations}

CEF: chicken embry fibroblast; DMEM: Dulbecco's modified Eagle's medium; GFP: green fluorescent protein; IFN: interferon; ISG: IFN-stimulated gene; MEF: mouse embryo fibroblast; RLU: relative light unit; RVFV: Rift Valley fever virus.

\section{Acknowledgements}

We thank Jovan Pavlovic for providing cells which were essential for this study. Work in the authors' laboratories is supported by the grants We 2616/ 5-2 from the Deutsche Forschungsgemeinschaft and $01 \mathrm{Kl} 0705$ of the Bundesministerium für Bildung und Forschung.

\section{Authors' contributions}

TK carried out the antiviral assays and MH generated the RVFV-Ren virus. TK, $\mathrm{MH}$ and FW designed the study. NP produced recombinant chIFN and prepared CEFs. TK, MH, and FW were responsible for drafting and finalizing the manuscript. All authors read and approved the manuscript.

\section{Competing interests}

The authors declare that they have no competing interests.

Received: 19 August 2009 Accepted: 26 February 2010

Published: 26 February 2010

\section{References}

1. Haller $\mathrm{O}$, Kochs $\mathrm{G}$, Weber $\mathrm{F}$ : The interferon response circuit: induction and suppression by pathogenic viruses. Virology 2006, 344:119-130.

2. Randall RE, Goodbourn S: Interferons and viruses: an interplay between induction, signalling, antiviral responses and virus countermeasures. Gen Virol 2008, 89:1-47.

3. Meager A: Biological assays for interferons. J Immunol Methods 2002, 261:21-36.

4. Uno K, Sato T, Takada Y, Fujioka K, Suginoshita Y, Kakimi K, Moriyasu F, Kishida T: A bioassay for serum interferon based on induction of $2^{\prime} 5^{\prime}$ oligoadenylate synthetase activity. J Interferon Cytokine Res 1998, 18:1011-1018.

5. Canosi U, Mascia M, Gazza L, Serlupi-Crescenzi O, Donini S, Antonetti F, Galli G: A highly precise reporter gene bioassay for type I interferon. $J$ Immunol Methods 1996, 199:69-76.

6. Francois C, Bernard I, Castelain S, Charleston B, Fray MD, Capiod JC, Duverlie G: Quantification of different human alpha interferon subtypes and pegylated interferon activities by measuring MxA promoter activation. Antimicrob Agents Chemother 2005, 49:3770-3775.

7. Lleonart $\mathrm{R}, \mathrm{Naf} \mathrm{D}$, Browning $H$, Weissmann $\mathrm{C}$ : A novel, quantitative bioassay for type I interferon using a recombinant indicator cell line. Biotechnology (N Y) 1990, 8:1263-1267.

8. Schwarz H, Harlin O, Ohnemus A, Kaspers B, Staeheli P: Synthesis of IFNbeta by virus-infected chicken embryo cells demonstrated with specific antisera and a new bioassay. J Interferon Cytokine Res 2004, 24:179-184.

9. Holzinger D, Jorns C, Stertz S, Boisson-Dupuis S, Thimme R, Weidmann M, Casanova $J$, Haller O, Kochs G: Induction of MxA gene expression by influenza A virus requires type I or type III interferon signaling. J Virol 2007, 81:7776-7785.

10. Ferreira $P C$, Peixoto ML, Silva MA, Golgher RR: Assay of human interferon in Vero cells by several methods. J Clin Microbiol 1979, 9:471-475.

11. Perler L, Pfister H, Schweizer M, Peterhans E, Jungi TW: A bioassay for interferon type I based on inhibition of Sendai virus growth. J Immunol Methods 1999, 222:189-196.

12. Martinez-Sobrido L, Giannakas P, Cubitt B, Garcia-Sastre A, de la Torre JC: Differential inhibition of type I interferon induction by arenavirus nucleoproteins. J Virol 2007, 81:12696-12703.

13. Munoz-Jordan JL, Laurent-Rolle M, Ashour J, Martinez-Sobrido L, Ashok M, Lipkin WI, Garcia-Sastre A: Inhibition of alpha/beta interferon signaling by the NS4B protein of flaviviruses. J Virol 2005, 79:8004-8013.

14. Newby CM, Sabin L, Pekosz A: The RNA binding domain of influenza A virus NS1 protein affects secretion of tumor necrosis factor alpha, interleukin-6, and interferon in primary murine tracheal epithelial cells. J Virol 2007, 81:9469-9480.

15. Billecocq A, Spiegel M, Vialat P, Kohl A, Weber F, Bouloy M, Haller O: NSs protein of Rift Valley fever virus blocks interferon production by inhibiting host gene transcription. J Virol 2004, 78:9798-9806.

16. Bouloy M, Janzen C, Vialat $\mathrm{P}$, Khun H, Pavlovic J, Huerre M, Haller O: Genetic evidence for an interferon-antagonistic function of rift valley fever virus nonstructural protein NSs. J Virol 2001, 75:1371-1377. 
17. Frese M, Kochs G, Feldmann H, Hertkorn C, Haller O: Inhibition of bunyaviruses, phleboviruses, and hantaviruses by human MxA protein. $J$ Virol 1996, 70:915-923.

18. Habjan M, Penski N, Wagner V, Spiegel M, Overby AK, Kochs G,

Huiskonen JT, Weber F: Efficient production of Rift Valley fever virus-like particles: The antiviral protein MxA can inhibit primary transcription of bunyaviruses. Virology 2009, 385:400-408.

19. Habjan M, Pichlmair A, Elliott RM, Overby AK, Glatter T, Gstaiger M, SupertiFurga $\mathrm{G}$, Unger $\mathrm{H}$, Weber F: NSs protein of rift valley fever virus induces the specific degradation of the double-stranded RNA-dependent protein kinase. J Virol 2009, 83:4365-4375.

20. Ikegami T, Narayanan K, Won S, Kamitani W, Peters CJ, Makino S: Rift Valley fever virus NSs protein promotes post-transcriptional downregulation of protein kinase PKR and inhibits elF2alpha phosphorylation. PLOS Pathog 2009, 5:e1000287.

21. Habjan M, Penski N, Spiegel M, Weber F: T7 RNA polymerase-dependent and -independent systems for CDNA-based rescue of Rift Valley fever virus. J Gen Virol 2008, 89:2157-2166.

22. Blakqori G, Delhaye S, Habjan M, Blair CD, Sanchez-Vargas I, Olson KE, Attarzadeh-Yazdi G, Fragkoudis R, Kohl A, Kalinke U, et al: La Crosse bunyavirus nonstructural protein NSs serves to suppress the type I interferon system of mammalian hosts. J Virol 2007, 81:4991-4999.

23. Schultz U, Kock J, Schlicht HJ, Staeheli P: Recombinant duck interferon: a new reagent for studying the mode of interferon action against hepatitis B virus. Virology 1995, 212:641-649.

24. Schultz U, Rinderle C, Sekellick MJ, Marcus PI, Staeheli P: Recombinant chicken interferon from Escherichia coli and transfected COS cells is biologically active. Eur J Biochem 1995, 229:73-76.

25. Barrett AD, Hunt N, Dimmock NJ: A rapid method for the inactivation of virus infectivity prior to assay for interferons. J Virol Methods 1984, 8:349-351.

doi:10.1186/1743-422X-7-50

Cite this article as: Kuri et al: Species-independent bioassay for sensitive quantification of antiviral type I interferons. Virology Journal 2010 7:50.

\section{Submit your next manuscript to BioMed Central and take full advantage of:}

- Convenient online submission

- Thorough peer review

- No space constraints or color figure charges

- Immediate publication on acceptance

- Inclusion in PubMed, CAS, Scopus and Google Scholar

- Research which is freely available for redistribution

Submit your manuscript at www.biomedcentral.com/submit 